\title{
Design and optimization of JFET region for high voltage 4H-SiC IGBT
}

\author{
Tongtong Yang ${ }^{\mathrm{a}}$, Runhua Huang and Song Bai \\ State Key Laboratory of Wide-Bandgap Semiconductor Power Electronic devices, Nanjing Electronic Devices \\ Institute, Nanjing, 210016, China
}

\begin{abstract}
The JFET region is an essential for IGBT devices. This paper mainly uses the Silvaco Atlas simulation tool to investigate the dependence of the characteristics of IGBT on JFET region. The simulations focus on the study of the width and concentration of JFET region at different interface charge densities. The results show that there is a paradox between the optimization of turn-on characteristics and blocking characteristics of IGBT devices. The best combinations of width and concentration at different charge densities are determined in considerations of both the turn-on and blocking characteristics. The simulation results show that a JFET width of $3 \mu \mathrm{m}$ and a doping concentration of $6 \mathrm{e} 15 \mathrm{~cm}^{-3}$ are the best combination in most situations for high voltage $(>20 \mathrm{kV})$ IGBT devices.
\end{abstract}

Keywords: JFET; 4H-SiC; IGBT.

\section{Introduction}

The $4 \mathrm{H}-\mathrm{SiC}$ material has more attractive properties than silicon including lower intrinsic carrier concentration and thermal generation rate, and higher breakdown electric field, which makes the operation of high voltage IGBT devices possible in harsh situations. IGBT is generally considered as an integration of MOS and bipolar device, which combines the best attributes of MOS and bipolar devices to achieve optimal device characteristics. The design of JFET region is vital for IGBT for the reason that various parameters of JFET region could significantly influence the device characteristics of IGBT devices. The simulation results in this paper show that a paradox exists in the design of JFET between the optimization of turn-on and blocking characteristics. For example, a longer width of JFET region is helpful in reducing the specific on resistances of IGBT, however, this will cause the device to break down at lower voltages. Therefore, the parameters of JFET must be optimized through the considerations on both of turn-on and blocking characteristics. In this paper, for the first time, the interface defect is also be considered and the influence on the change of best combination of width and concentration is presented. The paper is intended to view the changes of current density at various combinations of width and concentration of JFET region to determine the best combination at different interface charge densities. The simulations shows that if the interface charge density is lower than $1 \mathrm{e} 12 \mathrm{~cm}^{-2}$, the JFET width should be set to $3 \mu \mathrm{m}$, and the doping concentration should be kept at $6 \mathrm{e} 15 \mathrm{~cm}^{-3}$.

a Corresponding author: yangtongtong2010@163.com

(C) 2016. The authors - Published by Atlantis Press 


\section{Device structure}

The simplified designed cross-sections of $4 \mathrm{H}-\mathrm{SiC}$ N-IGBT is shown in Figure 1 . The device structure is optimized by the Atlas simulation tool. The vertical length between the edge of p-well and $n+$ region is about $1 \mu \mathrm{m}$, which could guarantee that the breakdown of device is not caused by the short vertical length even if the drain voltage is over $25 \mathrm{kV}$. The concentration distribution curve of p-well is also plotted in Figure 5, which is finally determined by the process simulation tool Athena. This paper is devoted to investigate high voltage $(>20 \mathrm{kV})$ devices, the drift layer thickness is then set to $190 \mu \mathrm{m}$ with uniform concentration of $2 \mathrm{e} 14 \mathrm{~cm}^{-3}$, which could make sure that the device is capable of blocking over $20 \mathrm{kV}$. The thickness and concentration of buffer layer is also optimized by author, and $5 \mu \mathrm{m}$ is chosen as the thickness and $3 \mathrm{e} 17 \mathrm{~cm}^{-3}$ as the uniform concentration. The channel length is approximately $1 \mu \mathrm{m}$. Compared to the resistance of the drift layer, the channel resistance could be nearly neglected.

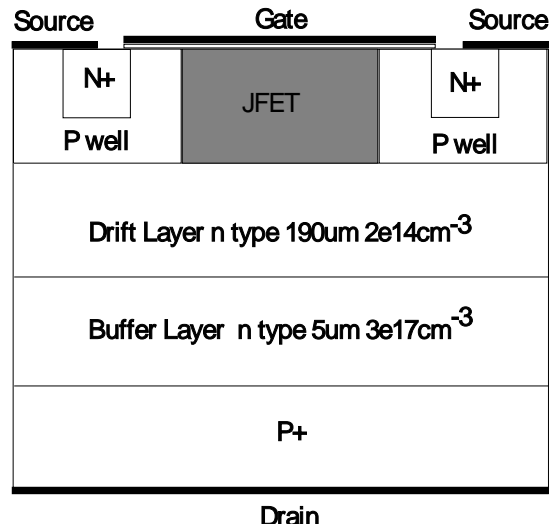

Figure 1. Simplified cross-section of N-IGBT

\begin{tabular}{l|l}
\hline Permittivity & 9.66 \\
Eg300 & $3.26 \mathrm{eV}$ \\
Egbeta & 0 \\
Egalpha & $3.2 \mathrm{e}-14$ \\
Taun0 for Drift Layer & $4.16 \mathrm{e}-6 \mathrm{~s}$ \\
Taup0 for Drift Layer & $4.16 \mathrm{e}-7 \mathrm{~s}$ \\
Taun0 for Buffer Layer & $1.1 \mathrm{e}-7 \mathrm{~s}$ \\
Taup0 for Buffer Layer & $1.1 \mathrm{e}-7 \mathrm{~s}$ \\
NSRHN & $3 e 17 \mathrm{~cm}^{-3}$ \\
NSRHP & $3 e 17 \mathrm{~cm}^{-3}$ \\
VSATN & $2 \mathrm{e} 14 \mathrm{~cm} / \mathrm{s}$ \\
VSATP & $2 \mathrm{e} 14 \mathrm{~cm} / \mathrm{s}$
\end{tabular}

Figure 2. Models parameters used in this paper

\section{Simulation results}

The Silvaco TCAD provides lots of physical models to make the simulations much more accurate. The different choice of models could directly influence the simulation results. Now 4H-SiC MOSFET is successfully fabricated in Nanjing Electronics devices Institute. And the blocking capability could be nearly $5 \mathrm{kV}$. Although there is much difference in the structures of MOSFET and IGBT, the simulation models adopted could be considered the same. Therefore, based on the real fabrication of MOS and the Atlas's Manual, we finally choose the models parameters described in Figure 2. 




(a)



(c)

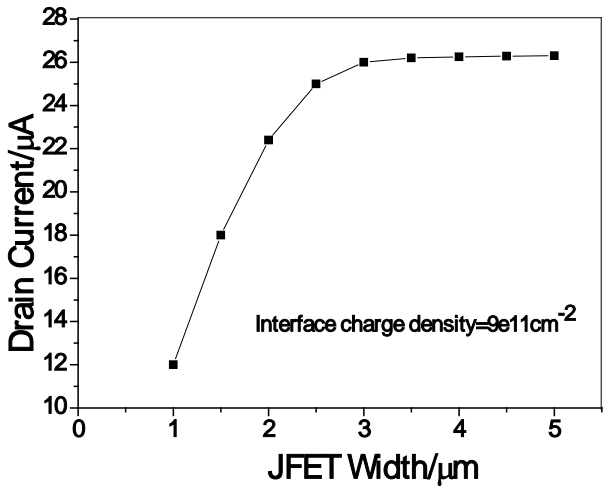

(e)

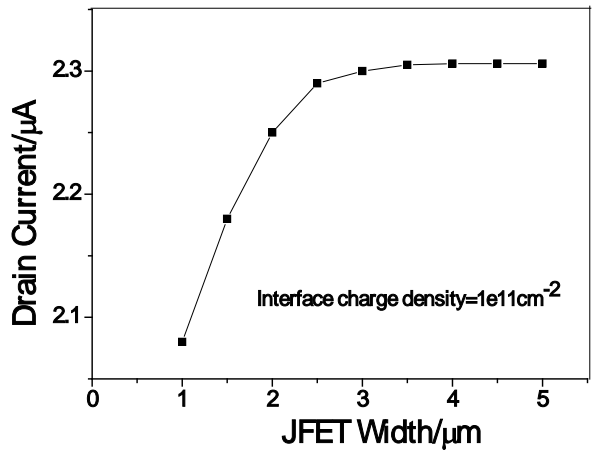

(b)

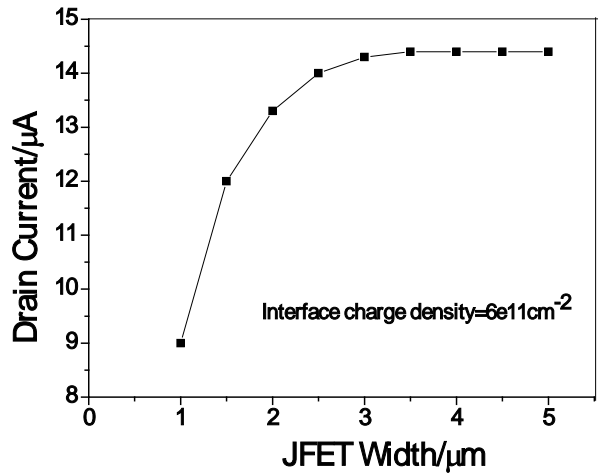

(d)

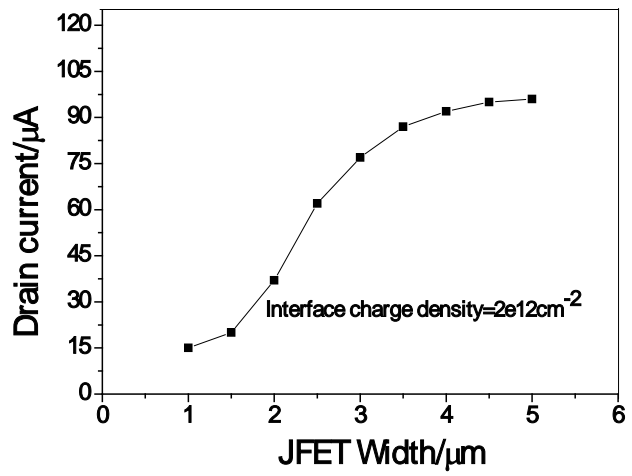

(f)

Figure 3. Dependence of drain current density on JFET width at different interface charge density
(a) $5 \mathrm{e} 10 \mathrm{~cm}^{-2}$
(b) $1 \mathrm{e} 11 \mathrm{~cm}^{-2}$
(c) $3 \mathrm{e} 11 \mathrm{~cm}^{-2}$
(d) $6 \mathrm{e} 11 \mathrm{~cm}^{-2}$
(d) $9 \mathrm{e} 11 \mathrm{~cm}^{-2}$
(f) $2 \mathrm{e} 12 \mathrm{~cm}^{-2}$

The relationship between drain current density and JFET width at various interface charge densities is clearly shown in Figure 3. The simulation results show that when the interface charge density is lower than $1 \mathrm{e} 12 \mathrm{~cm}^{-2}$, the drain current would reach a stable value at a JFET width of $3 \mu \mathrm{m}$ even if the charge density varies from $5 \mathrm{e} 10 \mathrm{~cm}^{-2}$ to $9 \mathrm{e} 11 \mathrm{~cm}^{-2}$. Therefore, in actual fabrication, if the interface charge density could be controlled lower than $1 \mathrm{e} 12 \mathrm{~cm}^{-2}$, a JFET length of $3 \mu \mathrm{m}$ would be welcomed by the designer to guarantee the blocking capability of IGBT.

The drain current increase as JFET width becomes larger. To explain the reason, a well-known formula should be introduced.

$$
\mathrm{R}=\rho \mathrm{L} / \mathrm{S}
$$


Here the $\mathrm{L}$ is vertical length of p-well or JFET region, a fixed value as mentioned above. In the device simulation, the width of the unit cell is set to $1 \mu \mathrm{m}$, so if we multiply this value by the JFET width, then we could get the "S" in formula (1). Therefore, if JFET width becomes larger, the "S" also increases, with a lower JFET resistance, causing the drain current to increase as well.

And the drain current also increases abruptly as the charge density becomes larger. IGBT works in a switching state at most time, so a larger drain current predicts larger turn-on losses, which is serious in high voltage applications. Maybe this is a reasonable explanation of pursuit of smooth interface in fabrication process.

However, when the charge density rises up to $2 \mathrm{e} 12 \mathrm{~cm}^{-2}$, the drain current continues increasing until the JFET width has a length of $5 \mu \mathrm{m}$, which should be paid much more attention. It tells us that when the charge density is higher than the level, the JFET length should be adjusted to a larger value to reduce the turn-on resistance while keeping expected blocking characteristics.

According to the fabrication of MOSFET, the interface charge density could be controlled at a level of about $3 \mathrm{e} 11 \mathrm{~cm}^{-2}$. So when we investigate the effect of concentration of JFET region, we fix the interface charge density to $3 \mathrm{e} 11 \mathrm{~cm}^{-2}$.

The concentration distribution of the p-well is also necessary to be considered carefully. As is well known, when the IGBT device is in a blocking state, the JFET region would be fully depleted finally to prevent the interface electric field from being too high. So the concentration of JFET region must vary in a safe range to make the depletion of the region not be difficult. The range should be considered according to the distribution curves of the P-well region. In this paper, the concentration of the p-well is determined based on the fabricated MOS device using the Atlas software.

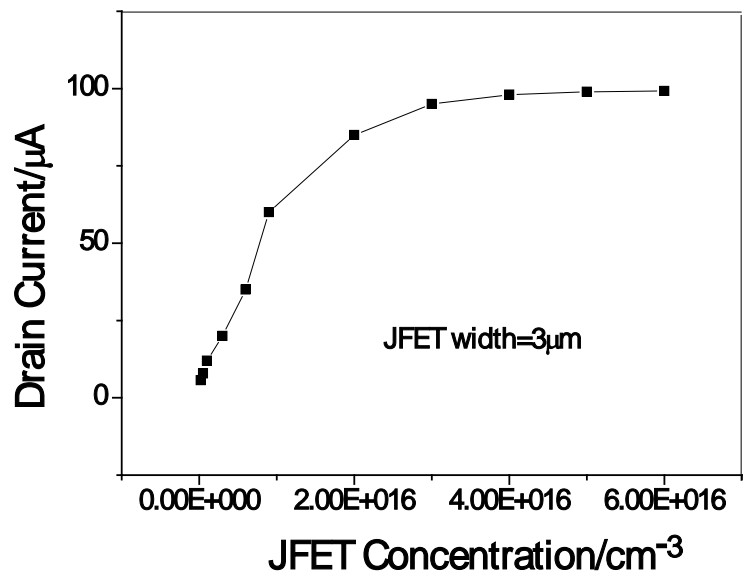

Figure 4. Dependence of drain current on JFET concentration

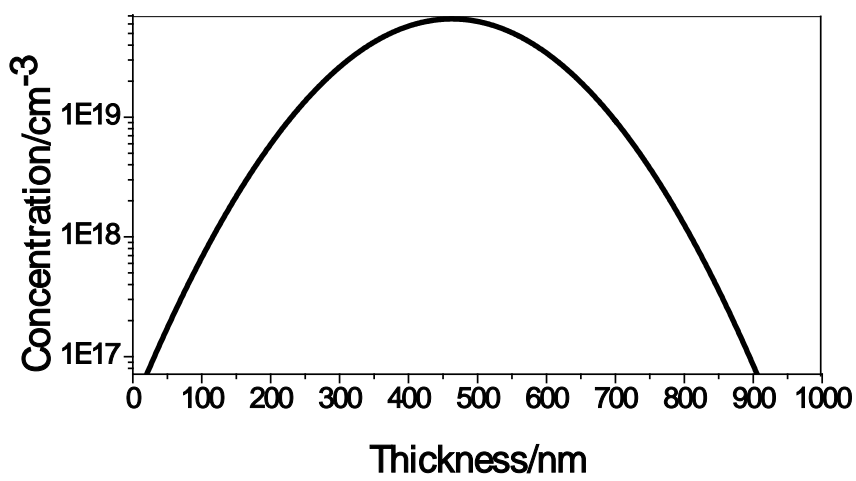

Figure 5. Concentration distribution curvesof the p-well 
Figure 4 shows the simulation result. The concentration varies from $2 \mathrm{e} 14 \mathrm{~cm}^{-3}$ to $6 \mathrm{e} 16 \mathrm{~cm}^{-3}$.As can be seen from the result, when the JFET concentration increases, the drain current increases as well. The result could be easily understood. Increasing the JFET concentration, then the JFET resistance will be much smaller, which causes the abrupt increase of drain current.

As is mentioned at the beginning of this paper, the optimization must be based on considerations of both turn-on characteristics and blocking characteristics. The blocking simulation result is shown in Figure 6.



Figure 6. Blocking characteristic when the JFET width varies from $1 \mu \mathrm{m}$ to $7 \mu \mathrm{m}$

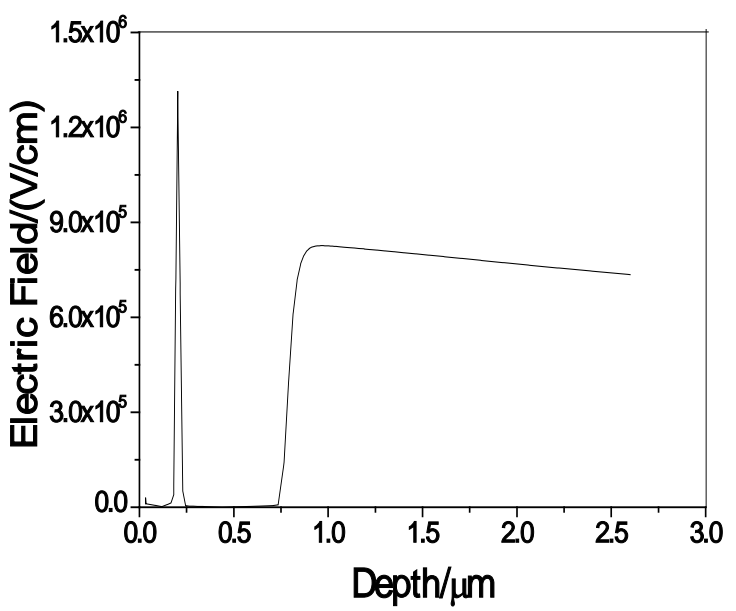

Figure 7. Electric field distribution from interface to bottom edge of p-well

As is shown in Figure6, when the JFET width increases, the breakdown voltage shows an abrupt decrease. Especially when the width varies from $3 \mu \mathrm{m}$ to $5 \mu \mathrm{m}$, the breakdown voltage decreases from the expected $22.6 \mathrm{kV}$ to about $5 \mathrm{kV}$. To explain this phenomenon, a distribution of electric filed from the interface to the bottom edge of the P-well is plotted in Figure 7.

It shows that, the interface electric filed become much larger than the reversed p-n junction. The result is consistent with the explanations mentioned previously. When the JFET width is larger than $3 \mu \mathrm{m}$, the JFET could not be easily fully depleted, which causes the JFET cannot efficiently prevent the interface electric field becoming too high. 


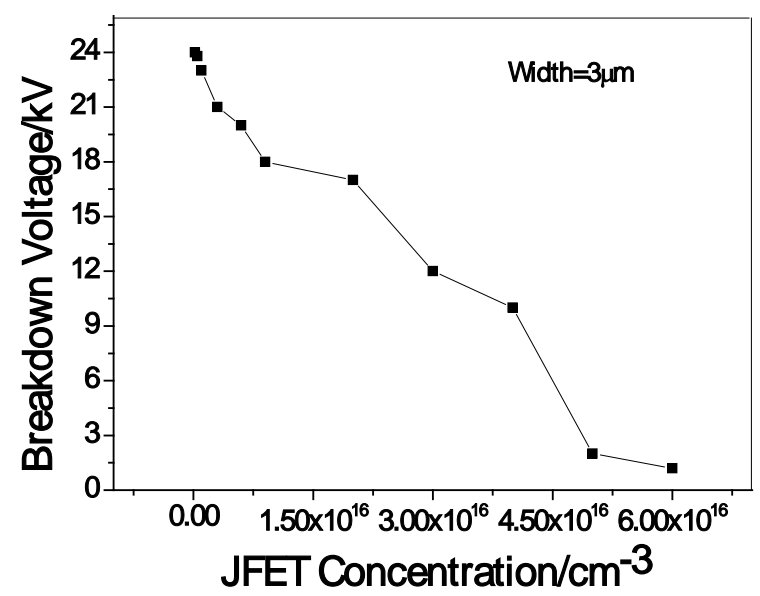

Figure 8. Blocking characteristics when the concentration varies from $2 \mathrm{e} 14 \mathrm{~cm}^{-3}$ to $6 \mathrm{e} 16 \mathrm{~cm}^{-3}$

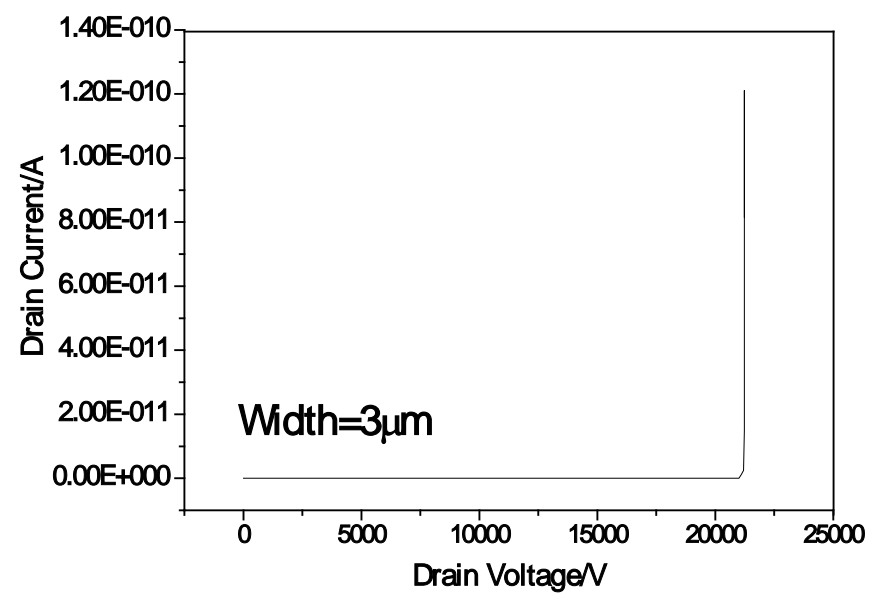

Figure 9. Blocking characteristics of IGBT when JFET width is $3 \mu \mathrm{m}$

When we discuss the turn-on characteristics, we finally find that when the JFET width is larger than $3 \mu \mathrm{m}$, the drain current reaches a nearly stable value, predicting a low turn-on resistance. And just as mentioned above, the blocking capability has an abrupt decrease when the JFET width is larger than $3 \mu \mathrm{m}$.Therefore, considering on both the turn-on and blocking characteristics, a JFET width of $3 \mu \mathrm{m}$ is almost the best.

Figure 8 shows the changes of breakdown voltage when the concentration varies from 2e14 to 6e16.The IGBT could still block over $18 \mathrm{kV}$ even if the doping concentration is higher than $2 \mathrm{e} 16 \mathrm{~cm}^{-3}$. When the concentration increases up to $6 \mathrm{e} 16 \mathrm{~cm}^{-3}$, the breakdown voltage is only about $1200 \mathrm{~V}$, which shows the uselessness of the device. To make the device have a blocking capability about $22 \mathrm{kV}$, the doping concentration should not be higher than $6 \mathrm{e} 15 \mathrm{~cm}^{-3}$, which could be seen from the picture.

To examine the efficiency of the optimization, we choose the JFET width of $3 \mu \mathrm{m}$ as the final width and a doping concentration of $6 \mathrm{e} 15 \mathrm{~cm}^{-3}$ as the best choice. Then the blocking simulation is shown in Figure 9. It could be clearly seen that the optimized device has a blocking capability of $22 \mathrm{kV}$, and the leakage drain current is quite low. 


\section{Conclusions}

All the simulation results considered, we could get that the width and doping concentration of JFET region could significantly influence both the turn-on characteristics and blocking characteristics. When the JFET width is longer than $3 \mu \mathrm{m}$, the blocking capability decreases abruptly. Also, when the doping concentration of the region is lower than $6 \mathrm{e} 15 \mathrm{~cm}^{-3}$, the blocking voltage is always higher than $22 \mathrm{kV}$. Therefore we can get that to make the device have the most attractive characteristics, a JFET width of $3 \mu \mathrm{m}$ should be set and a doping concentration level of $6 \mathrm{e} 15 \mathrm{~cm}^{-3}$ should be kept. However, the conclusion above is under the condition that the interface charge density is lower than $1 \mathrm{e} 12 \mathrm{~cm}^{-2}$. When restricted by the process technology, the smooth interface level not easy to reach, the JFET width should be longer than $3 \mu \mathrm{m}$. But the specific value of JFET width and doping concentration should be adjusted according to the detailed requirements.

\section{References}

1. M. Lee, A. Q. Huang, Solid-State Electronics 93,12(2014)

2. S.Ryu, C.Capell, C.Jonas, Materials Science Forum Vols 778-780,4(2014)

3. S.Ryu, C.Lin, D.Sarit, Materials Science Forum Vols 717-720,4(2012)

4. Lin Zhu, T.Paul.Chrow, Materials Science Forum Vols 527-529,4(2006)

5. S.Ryu, Materials Science Forum Vols 740-742,4(2013)

6. ATLAS User's Manual,www.silvaco.com 